ARTICLE

\title{
Structures of human dual oxidase 1 complex in low-calcium and high-calcium states
}

Jing-Xiang Wur,2,3, Rui Liu', Kangcheng Song ${ }^{1}$ \& Lei Chen (10) 1,2,3凶

Dual oxidases (DUOXs) produce hydrogen peroxide by transferring electrons from intracellular NADPH to extracellular oxygen. They are involved in many crucial biological processes and human diseases, especially in thyroid diseases. DUOXs are protein complexes co-assembled from the catalytic DUOX subunits and the auxiliary DUOXA subunits and their activities are regulated by intracellular calcium concentrations. Here, we report the cryo-EM structures of human DUOX1-DUOXA1 complex in both high-calcium and low-calcium states. These structures reveal the DUOX1 complex is a symmetric 2:2 hetero-tetramer stabilized by extensive inter-subunit interactions. Substrate NADPH and cofactor FAD are sandwiched between transmembrane domain and the cytosolic dehydrogenase domain of DUOX. In the presence of calcium ions, intracellular EF-hand modules might enhance the catalytic activity of DUOX by stabilizing the dehydrogenase domain in a conformation that allows electron transfer.

\footnotetext{
${ }^{1}$ State Key Laboratory of Membrane Biology, College of Future Technology, Institute of Molecular Medicine, Beijing Key Laboratory of Cardiometabolic Molecular Medicine, Peking University, 100871 Beijing, China. ${ }^{2}$ Peking-Tsinghua Center for Life Sciences, Peking University, 100871 Beijing, China.

${ }^{3}$ Academy for Advanced Interdisciplinary Studies, Peking University, 100871 Beijing, China. ${ }^{凶}$ email: chenlei2016@pku.edu.cn
} 
$\mathrm{R}$ eactive oxygen species (ROS) are oxygen-containing chemical species that are highly reactive, such as hydrogen peroxide and superoxide anion ${ }^{1}$. They participate in many physiological processes and are implicated in several pathological conditions ${ }^{1}$. ROS can be generated by a class of dedicated enzymes called NADPH oxidase (NOX) in a highly regulated manner. These enzymes are multi-pass transmembrane proteins that catalyze the reduction of extracellular or luminal oxygen by intracellular NADPH to generate superoxide anion or hydrogen peroxide. NOX proteins are involved in many biological processes, including host defense, differentiation, development, cell growth and survival, cytoskeletal reorganization, and modification of the extracellular matrix ${ }^{2}$.

Comprising the human NOX protein family are NOX1-5 and DUOX1-2 (ref. ${ }^{2}$ ). NOX2 protein catalyzes the production of superoxide anion during phagocytosis in neutrophils and is essential for host defense ${ }^{3}$. DUOX1-2 proteins are highly expressed in thyroid tissue and they catalyze the production of hydrogen peroxide, which is important for the biosynthesis of thyroid hormones ${ }^{4}$. The function of DUOX protein requires physical interactions with an auxiliary protein called dual oxidase maturation factor (DUOXA) ${ }^{5}$. DUOXA promotes the maturation and proper plasma membrane localization of DUOX ${ }^{5}$. DUOX protein is encoded by two homologous genes in human, namely DUOX1 and DUOX2. Similarly, DUOXA protein is encoded by DUOXA1 and DUOXA2. Loss-of-function mutations of DUOX2 or DUOXA2 in human cause congenital hypothyroidism ${ }^{6}$. Because of the important role of DUOX in thyroid tissue, they are also named thyroid oxidase ${ }^{4}$.

NOX family proteins share a common catalytic core, formed by a heme-coordinating transmembrane domain (TMD) and a cytosolic dehydrogenase $(\mathrm{DH})$ domain ${ }^{7}$. The $\mathrm{DH}$ domain binds intracellular substrate $\mathrm{NADPH}$ and cofactor $\mathrm{FAD}$, and shares sequence homology to the ferredoxin-NADP + reductase (FNR), which is composed of two subdomains ${ }^{8}$. In addition to the shared TMD-DH catalytic core of NOX, the functional DUOX protein has an additional large $\mathrm{N}$-terminal extracellular peroxidase homology domain (PHD) and a long intracellular loop 0 containing two EF-hand domains, and it requires an auxiliary DUOXA protein for proper function. The activity of DUOX is regulated by intracellular calcium concentration ${ }^{4}$. Prior to our studies, the structures of NOX family members are only available in the form of isolated domains, including the DH domain (PDB ID: 5O0X) ${ }^{9}$ and TMD (PDB ID: $5 \mathrm{O} 0 \mathrm{~T})^{9}$ of NOX5 from the algea Cylindrospermum stagnale (csNOX5) and a subdomain of human NOX2 DH domain (PDB ID: $3 A 1 F)$. Despite the functional importance of DUOX and other NOX family members, their structures in the context of full-length functional protein complex are still unknown. Several open questions for DUOX remain elusive: How is the DH domain engaged with TMD to perform the catalytic redox reaction? How does DUOXA protein interact and co-assemble with DUOX? How is the activity of DUOX regulated by intracellular calcium? To answer these fundamental questions, we sought to characterize DUOX-DUOXA protein complex both structurally and functionally. Here, we present the cryo-EM structures of human DUOX1-DUOXA1 (hDUOX1-hDUOXA1) complex in both highcalcium and low-calcium states, providing insights into the structure and mechanism of calcium activation for DUOX.

\section{Results}

Structure determination. To express the hDUOX1 protein, we constructed the matured hDUOX1 protein (20-1551) in frame with $\mathrm{N}$-terminal GFP tag guided by a rat $\mathrm{FSH} \beta$ signal peptide for efficient secretion ${ }^{10}$. The molecular weight of GFP-tagged hDUOX1 is $219 \mathrm{kDa}$. To monitor the formation of DUOX1-DUOXA1 complex, we fused the hDUOXA1 protein with a C-terminal MBP$\mathrm{mScarlet}$ tag to increase its molecular weight to $106 \mathrm{kDa}$. Fluorescence size-exclusion chromatography (FSEC) showed the coexpression of hDUOXA1 effectively shifted the peak of hDUOX1 toward higher molecular weight, suggesting the formation of a stable hDUOX1-hDUOXA1 hetero-complex (Fig. S1a, b). The peak positions indicated hDUOX1 migrated as a monomer, while hDUOX1-hDUOXA1 complex migrated as a heterotetramer (Fig. S1b). Moreover, we found the co-expression of DUOX1 and DUOXA1 resulted in cell membranes that showed robust calciumactivated, NADPH-dependent hydrogen peroxide production detected by the Amplex Red assay ${ }^{11}$ (Fig. 1a-c). In the low-calcium condition, DUOX1-DUOXA1 complex showed low basal activity (Fig. 1b, c). Addition of calcium not only reduced the $K_{\mathrm{m}}$, but also increased the $K_{\text {cat }}$ of DUOX1 complex, leading to the overall enhancement of enzymatic activity (Fig. 1b,c).

We solubilized and reconstituted hDUOX1-hDUOXA1 complex into peptidisc using $\mathrm{NSPr}^{12}$. Purified peptidisc sample showed a homogenous peak on SEC (Fig. S1c) and two major protein bands on SDS-PAGE, both of which could be trimmed upon PNGase F treatment (Fig. S1d), suggesting both of hDUOX1 and hDUOXA1 were modified by $\mathrm{N}$-linked glycosylation. UV-vis spectrum showed the peptidisc sample has characteristic Soret band with peak at $415 \mathrm{~nm}$ (Fig. S1e), indicating proper Fe (III) heme incorporation. Moreover, the highly purified peptidisc sample recapitulated the calcium-activated NADPH-dependent hydrogen peroxide production observed on membrane (Fig. S1f), confirming that the calcium-dependent activation is a built-in mechanism of hDUOX1-hDUOXA1 protein complex. However, we found the maximum activity of purified peptidisc sample was lower than the activity measured using crude cell membrane (Fig. $1 \mathrm{~b}$ and Fig. S1f), suggesting either membrane bilayer or endogenous lipids might play a role on DUOX activity. We prepared cryo-EM grids using the peptidisc sample, either in the presence of $2.5 \mathrm{mM}$ ethylene glycol tetraacetic acid (EGTA; low calcium) or $0.5 \mathrm{mM}$ free calcium (high calcium). Both samples contained $0.1 \mathrm{mM} \mathrm{FAD}$ as cofactor and $0.5 \mathrm{mM} \mathrm{NADPH}$ as substrate.

Single particle cryo-EM analysis showed the purified protein was homogeneous and showed twofold symmetry (Figs. S2-S4). The overall resolution of cryo-EM maps in the low-calcium and high-calcium states reached 2.7 and $2.6 \AA$, respectively (Table S1). The extracellular domains and TMD showed better local resolution than the cytosolic domains, suggesting the higher mobility of the cytosolic domains (Figs. S2g and S4g). To further improve the map quality of cytosolic domains, we exploited symmetry expansion ${ }^{13}$ and multibody refinement ${ }^{14}$ by dividing one protomer into the large body (the extracellular domain and TMD) and the small body (the cytosolic domains; Figs. S2c and S4c). The final resolutions of cytosolic domain reached 3.4 and $3.2 \AA$ for the low-calcium and high-calcium states, respectively (Figs. S2-4 and Table S1). The high map quality and available homology structures allowed us to build the order regions of the complex, which encompassed $88 \%$ of DUOX1 and $79 \%$ of DUOXA1 (Figs. S5-8 and Table S1). In the following text, we will focus on the high-calcium state structure unless noted otherwise, because of its higher resolution.

The architecture of hDUOX1-hDUOXA1 protein complex. hDUOX1 subunits and hDUOXA1 subunits co-assemble into a 2:2 heterotetrameric protein complex with molecular weight $\sim 457 \mathrm{kDa}$. The complex encompasses $140 \AA \times 105 \AA \times 160 \AA 3 \mathrm{D}$ space and has an overall twofold rotational symmetry (Fig. 1d-f). Vertically, the complex can be divided into three layers: the extracellular layer, the transmembrane layer, and the cytosolic 
a

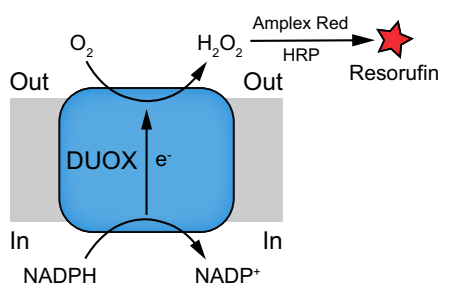

b

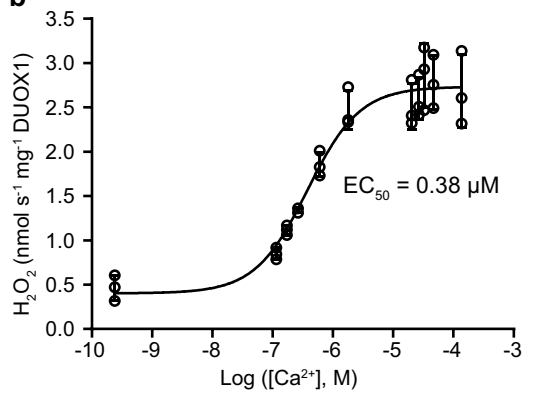

c

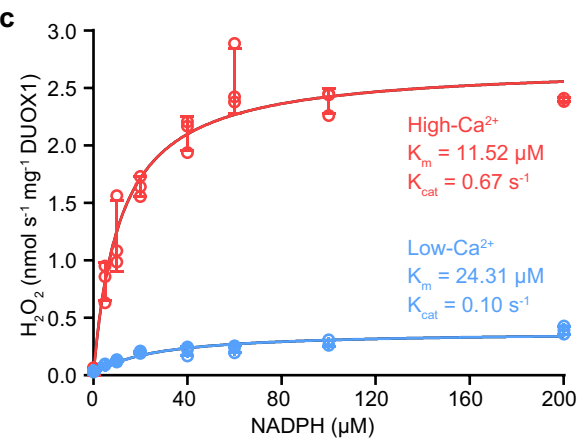

d

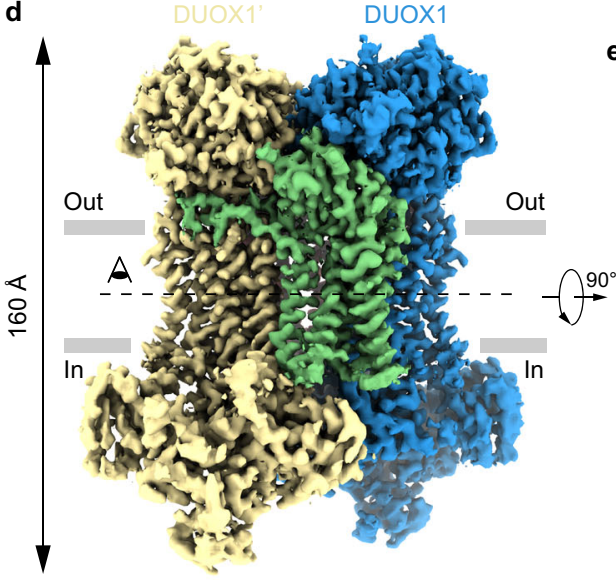

e

$\mathbf{f}$
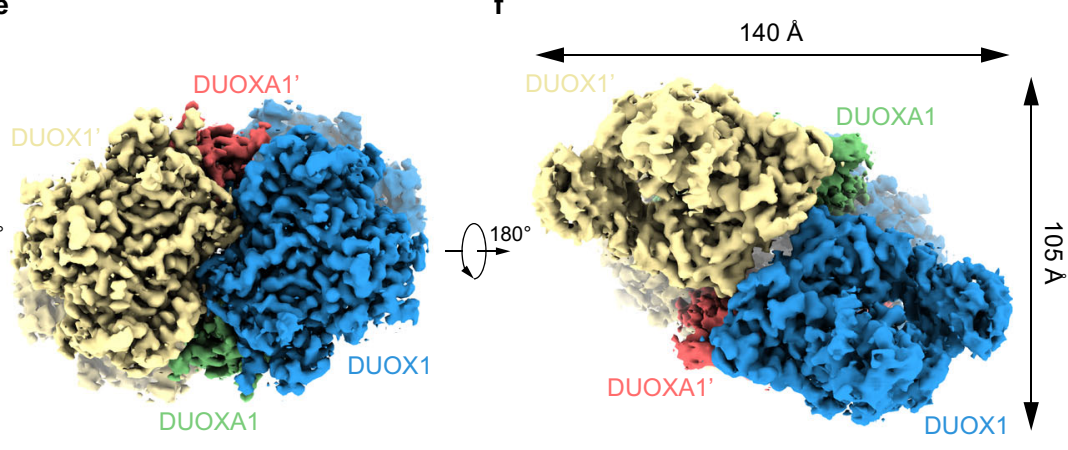

g

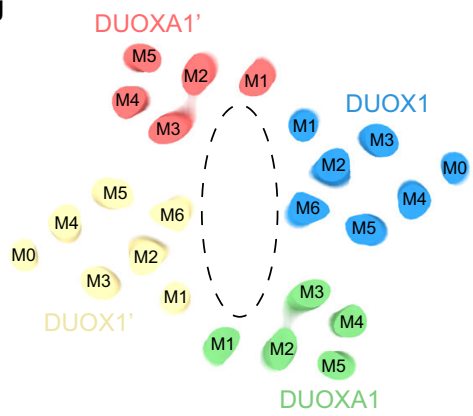

h

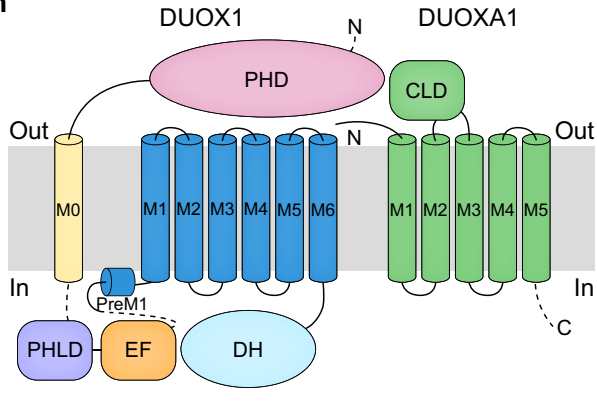

i

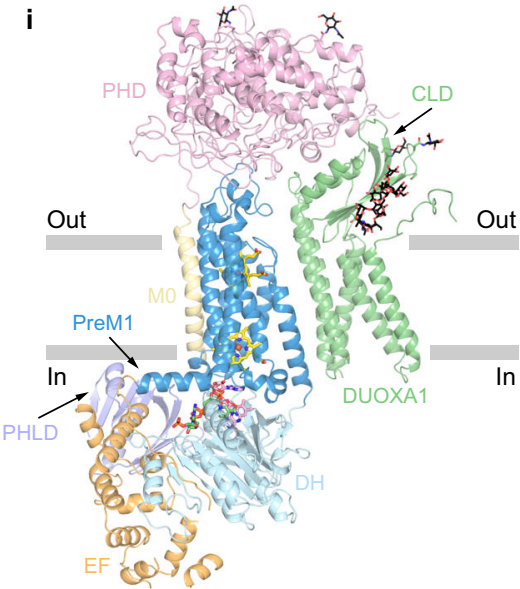

Fig. 1 Structure of human DUOX1-DUOXA1 complex in the high-calcium state. a Schematic of the DUOX enzymatic assay. In the presence of $\mathrm{H}_{2} \mathrm{O}_{2}$ (produced by DUOX), horseradish peroxidase (HRP) converts nonfluorescent Amplex Red to fluorescent resorufin, which is measurable and proportional to $\mathrm{H}_{2} \mathrm{O}_{2}$. b Calcium-dependent activation of hDUOX1-hDUOXA1 complex. Data are shown as means \pm standard deviations, $n=3$ biologically independent samples. Source data are provided as a Source data file. c Steady state enzyme activity of hDUOX1-hDUOXA1 complex as the function of NADPH concentration in the presence or absence of calcium. Data were fit to the Michaelis-Menten equation to obtain the $K_{\mathrm{m}}$ and $K_{\text {cat }}$ value. Data are shown as means \pm standard deviations, $n=3$ biologically independent samples. Source data are provided as a Source data file. $\mathbf{d}$ Side view of the cryo-EM map of hDUOX1-hDUOXA1 complex in the high-calcium state. The approximate boundaries of phospholipid bilayer are indicated as gray thick lines. One protomer of hDUOX1 and hDUOXA1 complex is colored as blue and green, the other one is colored as yellow and red, respectively. e A $90^{\circ}$ rotated top view compared to $\mathbf{d}$. $\mathbf{f ~ A ~} 180^{\circ}$ rotated bottom view compared to $\mathbf{e} . \mathbf{g}$ Top view of the cross-section of the transmembrane layer at the position indicated as a dashed line in $\mathbf{d}$. The large cavity in the transmembrane layer is indicated by dashed oval. For clarity, the cryo-EM map was low-pass filtered to $6 \AA$. h Topology of hDUOX1 and hDUOXA1 subunits. Transmembrane helices are shown as cylinders, unmodeled disordered regions are shown as dashed lines. The phospholipid bilayer is shown as gray layers. PHD peroxidase homology domain of hDUOX1, PHLD pleckstrin homology-like domain of hDUOX1, EF EFhand calcium-binding module of hDUOX1, DH dehydrogenase domain of hDUOX1, CLD claudin-like domain of hDUOXA1. i Structure of one protomer of hDUOX 1 and hDUOXA1 complex in cartoon representation. The colors of each individual domain are the same as in $\mathbf{g}$. The approximate boundaries of phospholipid bilayer are indicated as gray thick lines. Sugar moieties, hemes, FAD, and NADPH are shown as black, yellow, pink, and green sticks, respectively.

layer (Fig. 1d). In the extracellular layer, the two large N-terminal PHD domains of hDUOX1 pack against each other diagonally and are buttressed by the extracellular domain of DUOXA1 from beneath (Fig. 1d-f). The transmembrane layer is formed by 24 transmembrane helices and harbors the heme-binding sites that provide the electron transfer pathway across the membrane (Fig. 1g). At the center of the transmembrane layer, there is a large cavity without discernable protein densities. The interior 
surface of this cavity is highly hydrophobic (Fig. S3i) and there are several lipid molecules bound on this surface (Fig. S3i), suggesting this cavity is probably filled by phospholipids on the cell membrane. The cytosolic layer is comprised of the catalytic DH domain and regulatory domains for intracellular calcium sensing (Fig. 1f, i).

Structure of the catalytic hDUOX1 subunit. hDUOX1 is the catalytic subunit of the complex (Fig. 2). On the extracellular side of hDUOX1 resides the large N-terminal PHD domain which shares sequence homology with several peroxidases, such as peroxidase A from Dictyostelium discoideum (DdPoxA, PDB ID: 6ERC) ${ }^{15}$ (Fig. S9a). Functional peroxidases utilize histidinecoordinated heme as the cofactor for catalysis. However, key residues for heme binding, such as the heme ligand histidine, are missing in the PHD of hDUOX1. Indeed, we did not observe any heme density in the structure of hDUOX1 PHD, suggesting PHD is probably not enzymatic functional in term of peroxidase activity. Close inspection of the map reveals two putative cation densities in PHD. One cation (cation binding site 1, CBS1) is coordinated by the side chains of D397 and T332, and the main chain carbonyl groups of V399, T332, and R395 (Fig. S9b). The second cation (CBS2) is coordinated by the side chain of D109, D174, S176, and T170, and the carbonyl groups of T170 and W172 (Fig. S9b). We observed strong densities in these two sites in both low-calcium and high-calcium conditions (Fig. S9b), suggesting the bound cations might be sodium ions which were present in large quantities in our protein sample or calcium ions that bind very tightly. Both CBS1 and CBS2 are evolutionary conserved in DUOX (Fig. S5) and DdPoxA ${ }^{15}$ (Fig. S9c), indicating their functional importance. Interestingly, we found both CBS1 mutant (D397A + T332A) and CBS2 mutant (D109A + D174A) of DUOX1 failed to co-assemble with DUOXA1 (Fig. S9d). Because CBS1 and CBS2 are away from the subunit interfaces in the DUOX1-DUOXA1 complex, we speculate these mutants probably affect the folding of PHD domain, suggesting the role of CBS1 and CBS2 in protein stability.

The PHD packs on top of the TMD of DUOX1 through multiple noncovalent interactions (Fig. 2a). Moreover, a disulfide bond between C118 on PHD and C1165 on loop C of TMD further staples the bottom of PHD onto the top of TMD (Fig. 2a, d). In the TMD, hDUOX1 has an extra bent M0 helix at the periphery of M3 and M4, together with the canonical six TM helices of NOX protein family. M1-M6 of hDUOX1 form two heme-binding sites within the TMD. H1144 on M3 and H1238 on M5 coordinate the outer heme (Fig. 2b). H1130 on M3 and H1225 on M5 coordinate the inner heme (Fig. 2c). These four histidines are absolutely conserved in NOX family proteins (Fig. S6). We observed a spherical density surrounded by the invariant R1087 on M2, H1148 on M3, and outer heme-coordinating residue H1144 (Fig. 2b and Fig. S3c). Previous studies showed mutations of the csNOX5 residues corresponding to R1087 and H1148 of hDUOX1 affected the reoxidation of dithionite-reduced TMD by oxygen and this site was proposed to be the oxygen substrate binding site, namely oxygen-reducing center (Fig. S10a, b) ${ }^{9}$. Our structure observations in hDUOX1 support the hypothesis.

Preceding the M1 helix of DUOX1 TMD, an amphipathic preM1 helix floats on the inner leaflet of plasma membrane (Fig. 1h). This helix was previously observed in csNOX5 (ref. ${ }^{9}$ ) and is probably a shared feature of NOX family proteins. Between M0 and preM1 is a long cytosolic fragment loop 0. Cryo-EM maps reveal that the $\mathrm{N}$-terminal of loop 0 is a domain rich of $\beta$ sheets (Figs. S3g and S10c). Structural search using DALI server ${ }^{16}$ identified the $\beta$ sheets-rich domain is a crypto pleckstrin homology-like domain (PHLD) that shares little sequence homology, but high structural similarity to the $\mathrm{PH}$ domain proteins (Fig. S10c) ${ }^{17}$.

Following the PHLD, two EF-hand type calcium-binding domains (EF1 and EF2) form a compact helical module that is connected to the PHLD through aC (Fig. S6). Residues predicted to be responsible for calcium binding in EF1 and EF2 are evolutionary conserved in DUOX family proteins (Fig. S6). Although we did not observe the strong densities for small calcium ions due to poor local resolution (Figs. S2-3), the structure of EF-hand module closely resembles the small subunit of calcium-dependent protein phosphatase calcineurin in the calcium-bound state (PDB ID: 4IL1) ${ }^{18}$ (Fig. S10d), suggesting both EF1 and EF2 are loaded with calcium in the high-calcium state. Based on the homology structure (4IL1), side chains of D828, D830, N832, and E839 and the main chain carbonyl group of Y834 chelate one calcium ion in EF1 (Fig. S10e) and side chains of D864, D866, N868, E875, and the main chain carbonyl group of L870 chelate another calcium ion in EF2 (Fig. S10f). It is reported that mutations of any of these calcium-binding sites abolished calcium activation ${ }^{19}$ and E879K mutation in hDUOX2 (E875 in hDUOX1) leads to congenital hypothyroidism ${ }^{20}$, emphasizing their importance in calcium activation.

The C-terminal catalytic DH domain is connected to M6 of DUOX1 TMD via a short linker (Fig. 1h, i). The DH domain of hDUOX1 has a canonical DH fold and its structure is similar to csNOX5 (ref. 9; Fig.1i and Fig. S7). We observed strong densities for both FAD cofactor and NADPH substrate, and their binding sites were contributed from not only $\mathrm{DH}$ domain, but also TMD (Fig. 2h, i), as described later.

Inter-domain interactions in the high-calcium state. In the high-calcium state, individual domains of DUOX1 in the cytosolic layer are stabilized by multiple inter-domain interactions. The PHLD interacts with adjacent TMD and DH domains (Fig. 2e). The main chain carbonyl group of K653 on PHLD makes hydrogen bond with R1215 on loop D of TMD (Fig. 2e). Side chain of R674 of PHLD interacts with the main chain carbonyl group of E1348 and I1349 on al of the DH domain (Fig. 2f). The EF1-EF2 module in the high-calcium state shapes a crevice that embraces $a 4$ and post $a 4$ loop of the $\mathrm{DH}$ domain (Fig. $2 \mathrm{~g}$ and Fig. S10g). The interactions between the EF module and DH are mainly hydrophobic and involve F768, F772, F807, F819, F840, and F847 of the EF module, L1463, M1467, I1470, F1475, V1478, and F1484 of the DH domain (Fig. 2g). In addition, K814 of the EF module makes electrostatic interaction with E1281 on $\beta 2$ of DH (Fig. $2 \mathrm{~g}$ ). The interactions between the EF module and the $\mathrm{DH}$ domain of hDUOX1 mimic the interactions between calcineurin subunit $\mathrm{B}$ and $\mathrm{A}$ in the calcium-bound state (PDB ID: 4IL1) ${ }^{18}$ (Fig. S10g, h).

The linker between the EF module and preM1 helices binds in a groove on the surface of the $\mathrm{DH}$ domain (Fig. 1i). DH docks onto the bottom of TMD via polar interaction between R1270 on M6 and D1367 on $\beta 7$, and between R1113 on loop B of TMD and $\mathrm{N} 1550$ of DH (Fig. 2h, i). It is reported that R1111Q mutation in hDUOX2 (R1113 in hDUOX1) was identified in congenital hypothyroidism patients ${ }^{20}$, highlighting the importance of this inter-domain interaction. Moreover, both the FAD cofactor and NADPH substrate bind at the interface between DH and TMD. R1214 and R1131 in TMD form electrostatic interaction with phosphate of FAD. D1128 makes hydrogen bonding with ribose of FAD (Fig. 2h). E1039 and N1040 in TMD make hydrogen bonding with adenosine ring of NADPH, and R1036 make cation- $\pi$ interaction with both adenosine ring and electrostatic interaction with phosphate group of NADPH (Fig. 2i). Notably, R1495, R1424, and R1036 all participate in electrostatic 
a

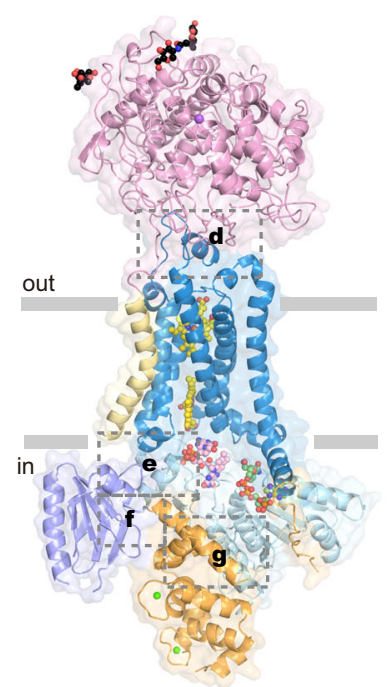

d

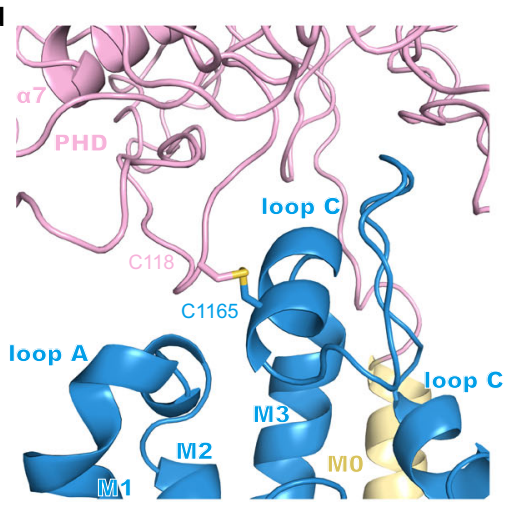

b

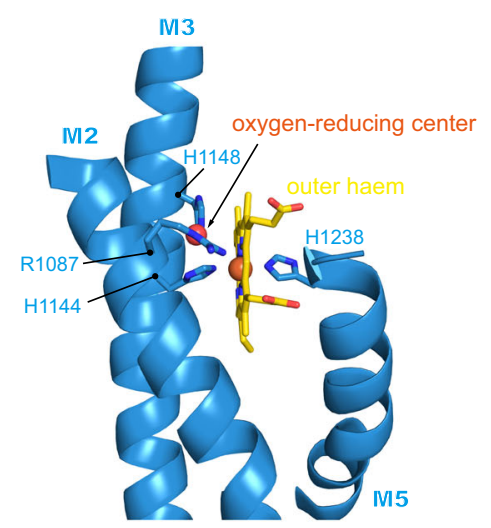

e

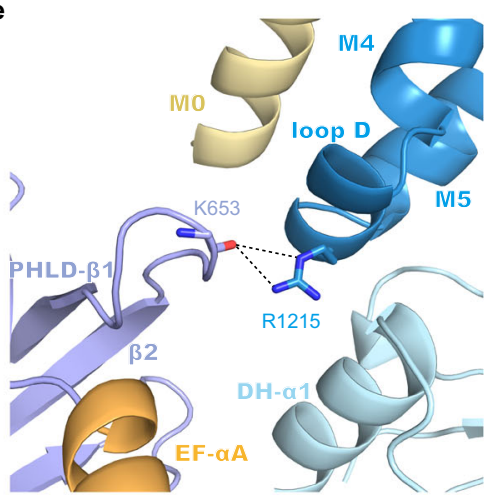

c

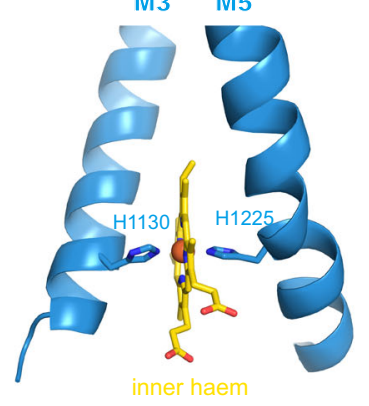

f

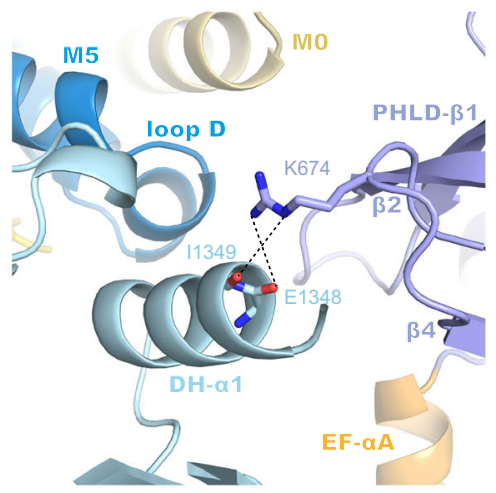

g

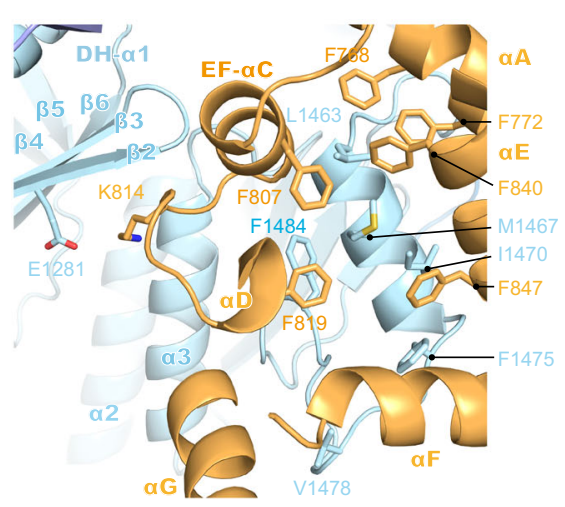

h

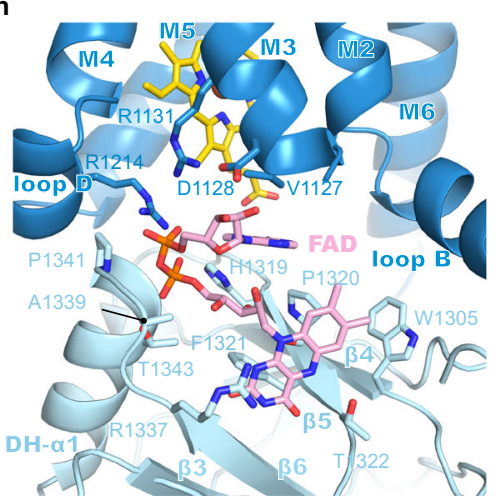

i

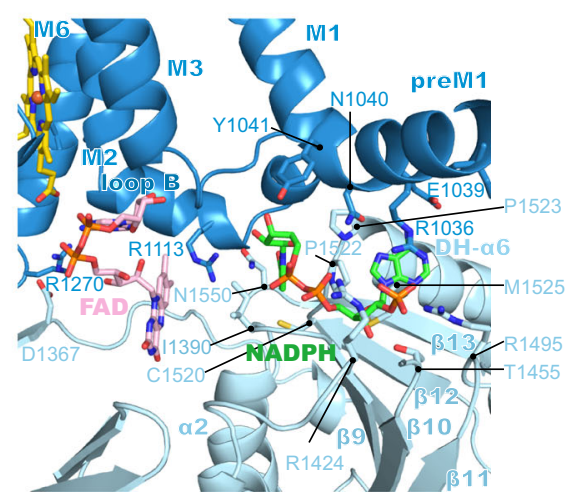

Fig. 2 Structure of hDUOX1 subunit. a Side view of hDUOX1 subunit in the high-calcium state, highlighting the key interfaces (boxed by dashed lines). Each domain is colored as in Fig. Th. The surface of hDUOX1 is shown in transparency. $\mathbf{b}$ The binding site of outer heme in the TMD. Heme is shown as sticks and colored in yellow. Unrelated helices in TMD are omitted for clarity. The putative oxygen-reducing center is indicated by arrow. c The binding site of inner heme in the TMD. $\mathbf{d}$ The interface between PHD and TMD boxed in a. Disulfide bond between C118-C1165 is shown as golden sticks. e The interface between PHLD and TMD boxed in $\mathbf{a}$, the hydrogen bonds are indicated with dashed lines. $\mathbf{f}$ The interface between PHLD and DH domain. $\mathbf{g}$ The interface between EF module and DH domain. $\mathbf{h}$ The FAD-binding site located at the interface between TMD and DH domain. Ligands and interacting residues are shown as sticks. $\mathbf{i}$ The NADPH-binding site located at the interface between TMD and DH domain.

interactions with the phosphate group of NADPH ribose, providing structural mechanism to distinguish NADPH from NADH (Fig. 2i). Through structural comparison, we found the NADPH-binding site in the csNOX5 structure was blocked by the artificially engineered C-terminal insertion, which was introduced into previous crystallization construct ${ }^{9}$ (Fig. S10i). Moreover, the adenosine group of $\mathrm{FAD}$ has a $180^{\circ}$ flip compared with structure of the isolated DH of csNOX5 (Fig. S10j). This is probably because D1128 on TMD stabilizes the ribose of FAD in such a conformation to make the connecting adenosine group of FAD in close proximity with inner heme for electron transfer (Fig. 2h). Taken together, the binding of FAD and NADPH at the interface 

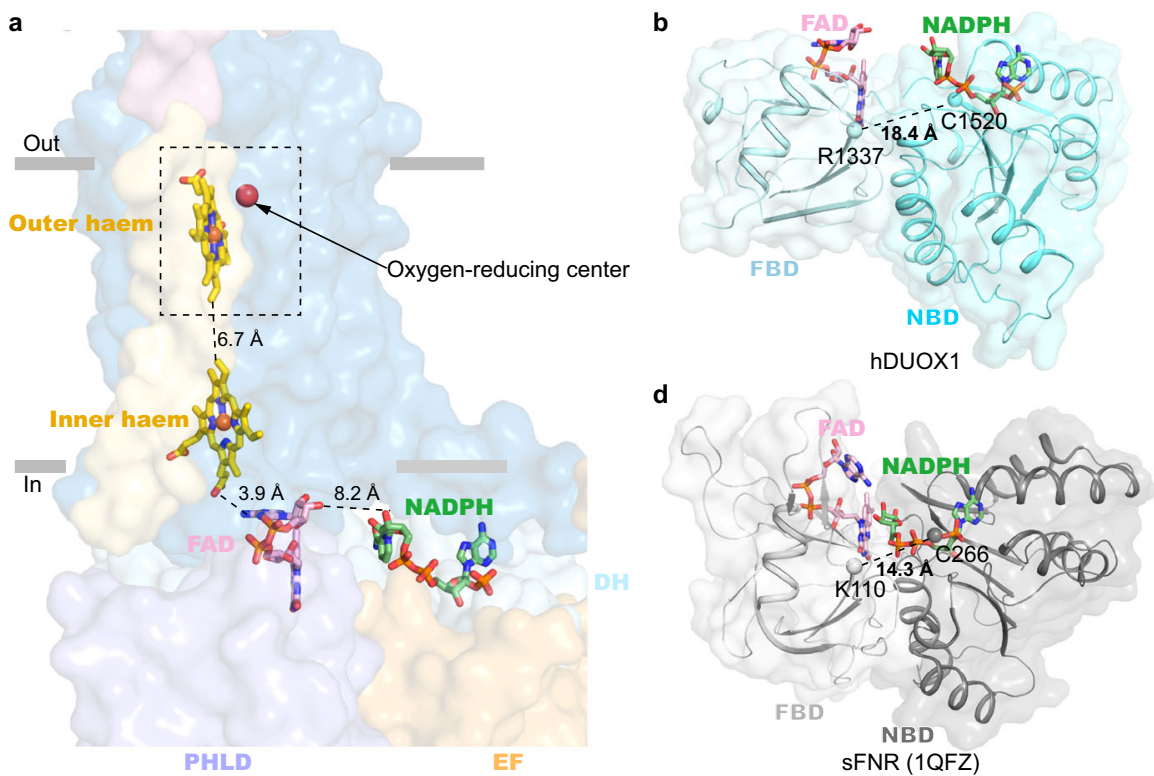

f

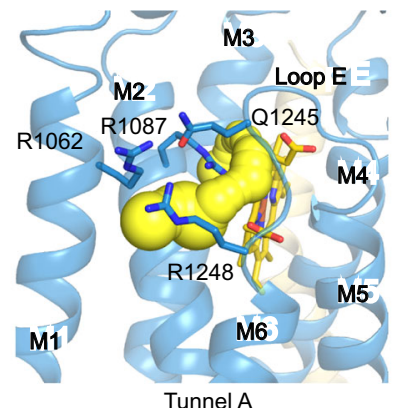

g

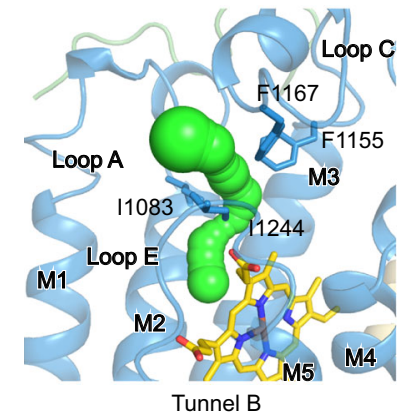

h

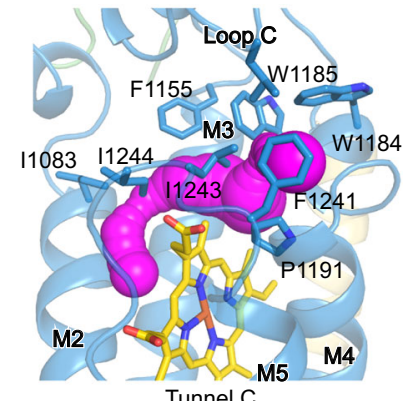

i

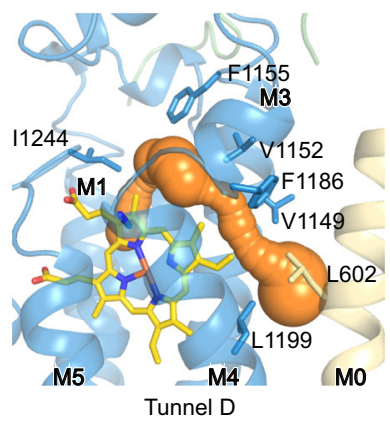

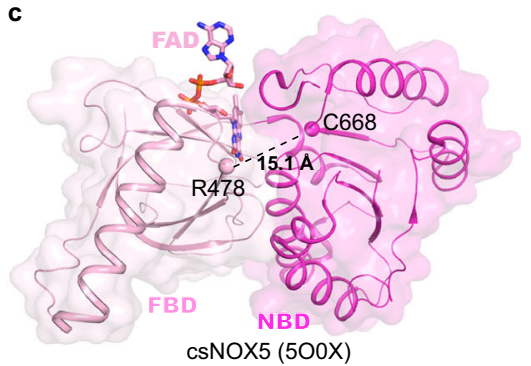

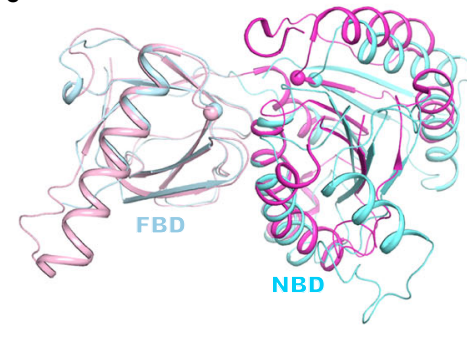

hDUOX1 vs csNOX5

j

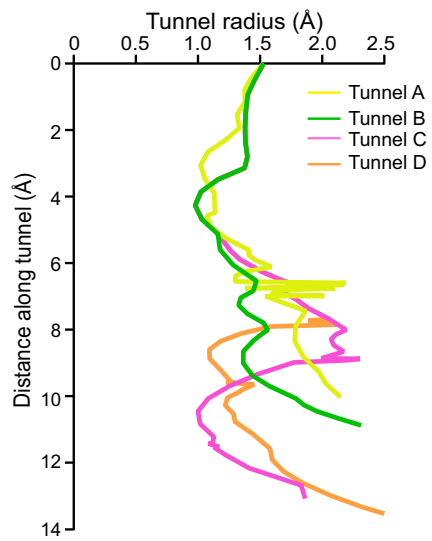

Fig. 3 Electron transfer pathway in hDUOX1 subunit in the high-calcium state. a The edge-to-edge distances between NADPH and FAD, FAD and inner heme, and two hemes are shown beside dashes. The ligands are shown as sticks, each domain of hDUOX1 are shown in surface, and colored the same as Fig. 1h. Only one hDUOX subunit is shown for clarity. The putative oxygen-reducing center is boxed by dashed lines. b-e The DH domain of hDUOX1 in a relaxed conformation (b), DH domain of $\operatorname{csNOX5}(\mathbf{c})$, and sFNR (d) in a tense conformation. The ligands are shown as sticks, two subdomains (FADbinding domain, FBD, and NADPH-binding domain, NBD) of DH are shown as cartoon with surface. Distances between C $\alpha$ atoms of the Arg (Lys in sFNR) of FBD and the Cys of NBD (shown as spheres) are labeled. e Structural comparison of the hDUOX1 DH domain (cyan) and csNOX5 (purple). FBD is used for structural alignment. $\mathbf{f - i}$ The close-up view of the putative oxygen-reducing center. Four predicted tunnels for oxygen substrate entrance and product exit are shown as surface in yellow, green, magenta, and orange, respectively. Residues surrounding the tunnels are shown as sticks. $\mathbf{j}$ Calculated radii of tunnels shown in $\mathbf{f}-\mathbf{i}$. The putative oxygen-reducing center is used as the starting point for calculation.

between DH and TMD of hDUOX1 would stabilize the docking of $\mathrm{DH}$ onto the bottom of TMD.

The putative electron transfer pathway. The measured edge-toedge distances between NADPH and FAD, between FAD and inner heme, and between inner heme and outer heme are 8.2, 3.9, and $6.7 \AA$, respectively (Fig. 3a). Although, it is possible that there are additional protein residues on DUOX1 that rely electrons from NADPH to FAD, such as W378 between two hemes in
csNOX5 (ref. ${ }^{9}$ ), the distance between NADPH and FAD is larger than that in the canonical FNR protein, such as $3.2 \AA$ in spinach FNR (sFNR, PDB ID: 1QFZ) ${ }^{21}$. Through structural comparison, we found the DH domain of DUOX1 shows a relaxed conformation, in which two subdomains are loosely packed, while both the DH of csNOX5 and sFNR show a tense conformation and their two subdomains are tightly packed against each other to bring FAD and NADPH into close proximity for electron transfer (Fig. $3 \mathrm{~b}-\mathrm{e}$ ). Therefore, the electron transfer efficiency in the current structure of DUOX1 is not optimal. Because the DUOX1 

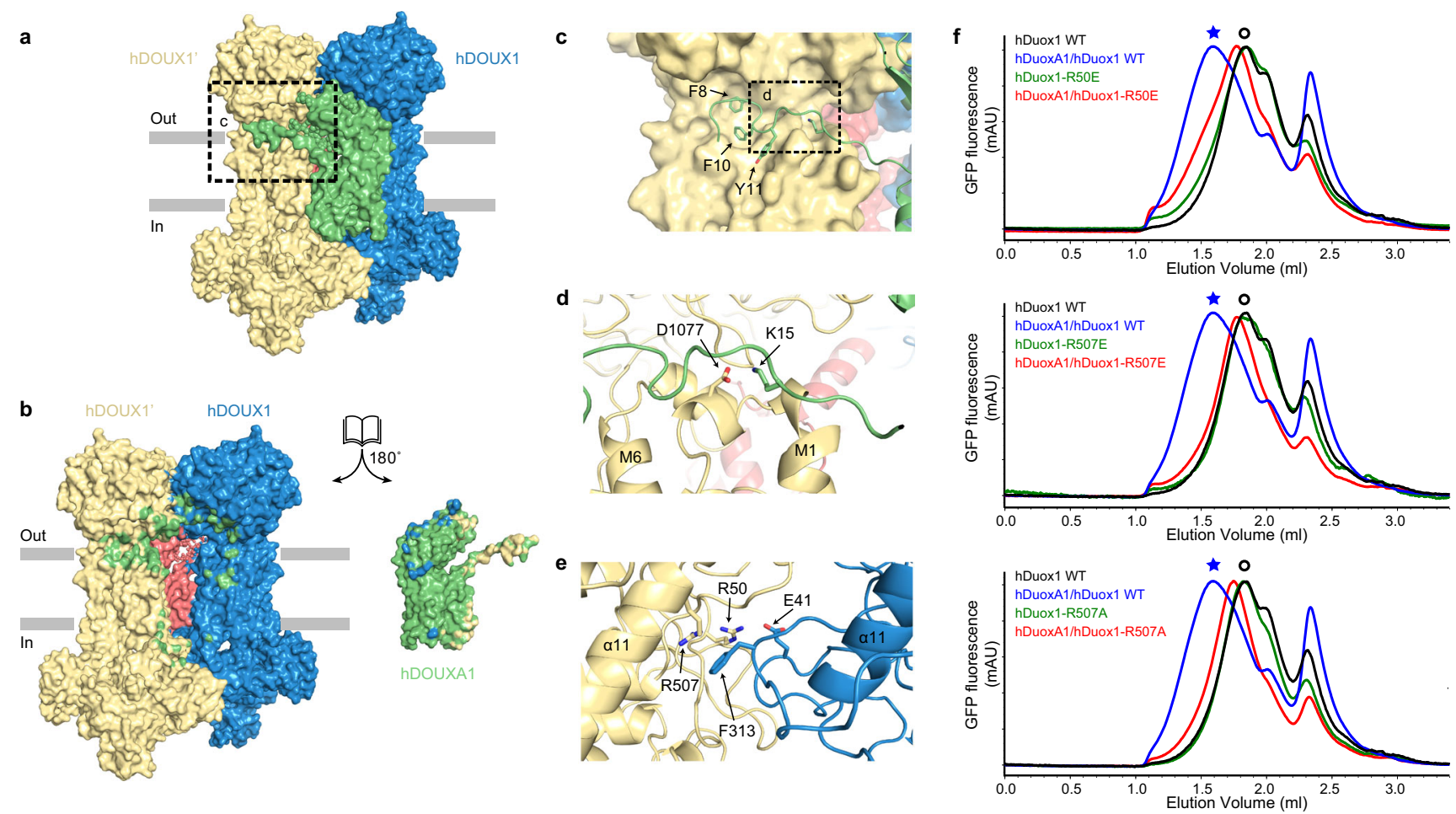

Fig. 4 Mechanism of hDUOX1-hDUOXA1 tetramer assembly. a The side view of hDUOX1-hDUOXA1 protein complex shown in surface representation and colored the same as in Fig. 1d. $\mathbf{b}$ The open-book view of the inter-subunit interfaces. Residues of hDUOX1 subunits that interact with hDUOXA1 subunit are colored in green. Residues of hDUOXA1 subunit that interact with hDUOX1 subunits are colored in yellow and blue. c The close-up view of the interactions between NTP of hDUOXA1 and hDUOX1 boxed in $\mathbf{a}$. $\mathbf{d}$ The close-up view of additional interactions between NTP of hDUOXA1 and hDUOX1 boxed in c. e The top view of interactions between PHD of two opposing hDUOX1 subunits. $\mathbf{f}$ Representative FSEC traces of hDOUX1 R50E, R507E, and R507A mutants are compared to that of wild-type (WT) hDOUX1. The peak position of the hDOUX1 peak is denoted by the hollow circles. Asterisks denote the peak position of hDUOX1-hDUOXA1 protein complex.

complex on cell membrane exhibited higher activity (Fig. $1 \mathrm{~b}$ and Fig. S1f), it is possible that lipids on cell membrane or the bilayer environment could somehow affect the structure of DUOX1 to enhance its electron transfer efficiency.

At the terminus of electron transfer chain near the extracellular side, the initial product of oxygen-reducing reaction is superoxide anion. We probed the possible pathways for oxygen entrance and for superoxide anion exit with CAVER ${ }^{22}$, using the oxygenreducing center as the starting point. We located four possible tunnels: tunnel A is formed by M1, M2, M5, and M6 and is capped by loop E on top (Fig. 3f); tunnel B is surrounded by M2, loop A, loop C, and loop E (Fig. 3g); tunnel C is embraced by M3, M4, and loop C (Fig. 3h); and tunnel D is enclosed by M3, M4, loop C, and M0 (Fig. 3i). The bottleneck radii of these tunnels are $\sim 1 \AA$ (Fig. 3j), which may allow the permeation of small oxygen substrate under dynamic motion of DUOX1 protein. Further analysis showed tunnels B-D are all surrounded by hydrophobic residues (Fig. 3g-i), which are unfavorable for superoxide anion permeation. In contrast, tunnel A is gated by hydrophilic R1087 on M2, R1062 on M1, and R1248 and Q1245 on loop E (Fig. 3f). We speculate the highly positively charged constriction of tunnel A would strongly attract the negatively charged superoxide anions, and this might be essential for the dismutation reaction between two superoxide anions to generate uncharged hydrogen peroxide for diffusion. Therefore, manipulations that may alter the constrictions of tunnels A-D would affect superoxide anion intermediate leakage. Indeed, it is reported that mutations on DUOX1 loop A or on DUOXA1 N-terminus peptide (NTP) which interacts with and stabilizes loop A would change the ratio of superoxide anion and hydrogen peroxide produced, probably by affecting the leakage of superoxide anions through these tunnels ${ }^{23-25}$.

Structure of hDUOXA1 and mechanism of complex assembly. DUOXA protein is an essential auxiliary subunit for DUOX enzyme $^{5}$ (Fig. S11a) and it has an extracellular N-terminus that is important for hydrogen peroxide generation ${ }^{24,25}$. We observed the NTP of hDUOXA1 extends and packs onto the PHD-TMD junction of the distal hDUOX1 subunit (Fig. 4a-e). Side chains of F8, F10, and Y11 of NTP insert into the hydrophobic groove formed by loop C, loop A, and PHD of hDUOX1 (Fig. 4c). In addition, K15 of DUOXA1 NTP makes electrostatic interactions with D1077 of DUOX1 (Fig. 4d). This agrees with previous data showing DUOXA1 NTP interacts with DUOX1 loop A (ref. ${ }^{23}$ ). hDUOXA1 has five transmembrane helices. Lower part of TM1 interacts with preM1 and M1 of hDUOX1 (Fig. S11a). The remaining four helices and associated extracellular loops share structural similarity with claudin superfamily members, such as claudin-9 (PDB ID: 6OV2) ${ }^{26}$ (Fig. S11). The extracellular loop between TM2 and TM3 folds into a compact claudin-like domain (CLD) composed of four $\beta$ strands and two $\alpha$ helices (Figs. S8 and S11). CLD forms extensive interactions with both distal and proximal DUOX1 subunits (Fig. 4a, b), emphasizing its important role in the complex assembly. This agrees with previous studies showing that splicing variants at TM2-TM3 loop have distinct behavior in supporting the activity of DUOX1 (ref. ${ }^{24}$ ). Moreover, we found an ordered N-linked glycosylation decoration on N109 of hDUOXA1 and its branched sugar moieties make extensive polar interactions with both DUOXA1 and DUOX1 subunits 
a

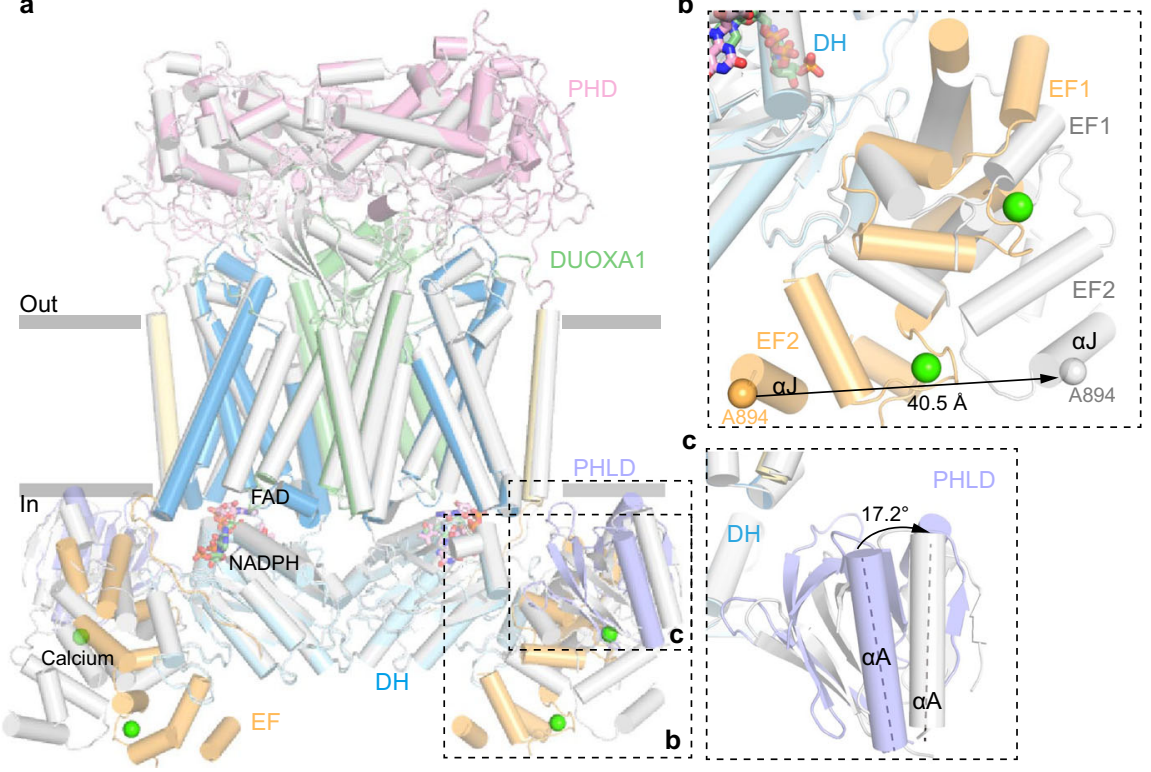

d

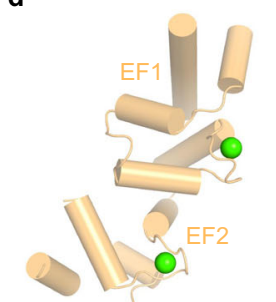

high-calcium state

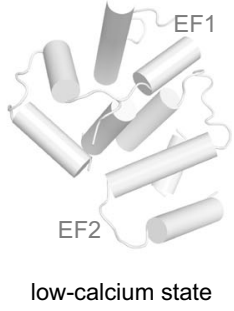

f

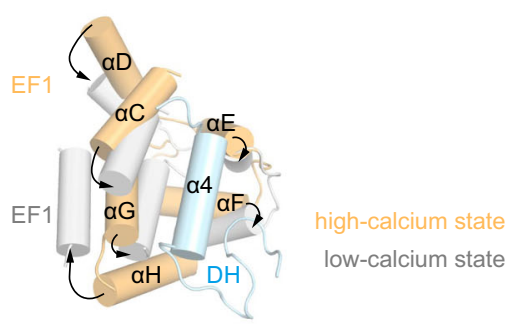

Fig. 5 Conformational change of hDUOX1 complex during calcium activation. a Structural comparison of hDUOX-hDUOXA1 complex between the highcalcium state (colored) and the low-calcium states (gray). Protein is shown as cartoon. Regions with large conformational changes are boxed by dashed lines. $\mathbf{b}$ Close-up view of the conformational changes of EF-hand module. C $\alpha$ atom of A894 on $\alpha$ J helix is used as marker to measure the movement of EF2. c Close-up view of the conformational change of PHLD. The angle between $\alpha A$ helices in the high-calcium and low-calcium states was measured. d, e Conformational differences of EF-hand module between the high-calcium state and the low-calcium state. $\mathbf{f}$ Reconfiguration of the interface between EF-hand module and $\alpha 4$ helix of DH domain. Arrows denote movements from high-calcium state into the low-calcium state.

(Fig. 4a, b). The PHD of two DUOX1 subunits also interact with each other (Fig. 4a, b). Close to the dyad axis, R50 and R507 on one PHD make polar interactions with E41 and F313 on the opposite PHD (Fig. 4e). We further analyzed the effects of interface mutations on the tetramer assembly, and found mutations of R50E, R507E, and R507A all severely affect tetrameric peak formation on FSEC (Fig. 4f). These structural information and biochemical data revealed the detailed inter-subunit interactions that dictate the heterotetramer assembly.

\section{Conformational change of DUOX1 complex upon calcium} activation. The consensus map in the low-calcium state showed the cytosolic layer had poor local resolution, which was improved by multibody refinement ${ }^{14}$ (Fig. S4). Further molecular flexibility analysis ${ }^{14}$ showed the cytosolic domains (small body) in the lowcalcium state were sampling a broad range of orientations relative to the TMD, evidenced by the plateau-shaped distribution on the histogram of the major eigenvector (Fig. S4f). This is in great contrast to the normal distribution in the high-calcium state (Fig. $\mathrm{S} 2 \mathrm{f}$ ), suggesting the cytosolic layer in the low-calcium state is more flexible. We compared the structures in the low-calcium state and high-calcium state, and found structural changes in the extracellular layer and transmembrane layer are small (Fig. 5a). However, there are large conformational changes of the regulatory PHLD and EF-hand module in the cytosolic layer (Fig. 5a-c and Movie S1). In the absence of calcium, the EF module switches from an extended shape into a more contracted shape (Fig. 5d, e), which reconfigures the interface between the EF module and a 4 of the $\mathrm{DH}$ domain, resulting in a loosely packed structure (Fig. 5f). In the low-calcium state, EF2 moves away from the $\mathrm{DH}$ domain. The Ca atom of A894 on aJ of EF2 has $40 \AA$ displacement (Fig. 5b). PHLD rotates away from the TMD and $\mathrm{DH}$ domains, and $\alpha \mathrm{A}$ of PHLD has $17.2^{\circ}$ outward rotation (Fig. 5c). As a result, several inter-domain interactions observed in the high-calcium state were disrupted and therefore the docking of $\mathrm{DH}$ domain onto $\mathrm{TMD}$ is weakened by these structural changes, leading to a higher mobility of $\mathrm{DH}$ domain (Fig. S4g). We propose the increased mobility of $\mathrm{DH}$ domain negatively correlates with the electron transfer efficiency and thus the catalytic activity of DUOX. In addition, because TMD also contributes to FAD and NADPH binding, the increased mobility of the DH domain would result in the reduced affinity of NADPH as well. This is in agreement with the markedly reduced $K_{\text {cat }}$ and moderately increased $K_{\mathrm{m}}$ in the low-calcium state, as we observed (Fig. 1c).

During the preparation of this manuscript, another group reported the structures of mouse DUOX1-DUOXA1 complex ${ }^{27}$. Interestingly, they found mouse DUOX1 complex exists in both heterodimeric and heterotetrameric form, and they proposed the activity of DUOX1 complex is regulated by dimer-tetramer assembly $^{27}$. This is in contrast to our observation that majority of hDUOX1 complex is in a homogenous tetrameric form (Fig. S1c). Whether this difference is due to different protein preparation procedure or different species (mouse vs human) remain elusive. Moreover, the intracellular PHLD and EF domains were not resolved in mouse DUOX1 complex structure because of insufficient map quality ${ }^{27}$. The overall structures of resolved parts between mouse and hDUOX1 complex are similar with root mean square deviation of 1.521 and $0.908 \AA$ for DUOX1 and DUOXA1 subunit, respectively (Fig. S11c). However, detailed structural comparison revealed several differences especially in the atomic models of FAD (Fig. S11d) and NADPH (Fig. S11e), probably due to the poor local map quality of mouse DUOX1 complex (EMD-21964).

\section{Discussion}

In this study, we provided the structures of hDUOX1-hDUOXA1 as a peptidisc-stabilized heterotetrameric protein complex in both high-calcium and low-calcium states. The structure of hDUOX1 complex in the high-calcium state reveals multiple inter-domain interactions that orientate DH and TMD for electron transfer, and thus redox reaction. Removal of calcium ions results in the 

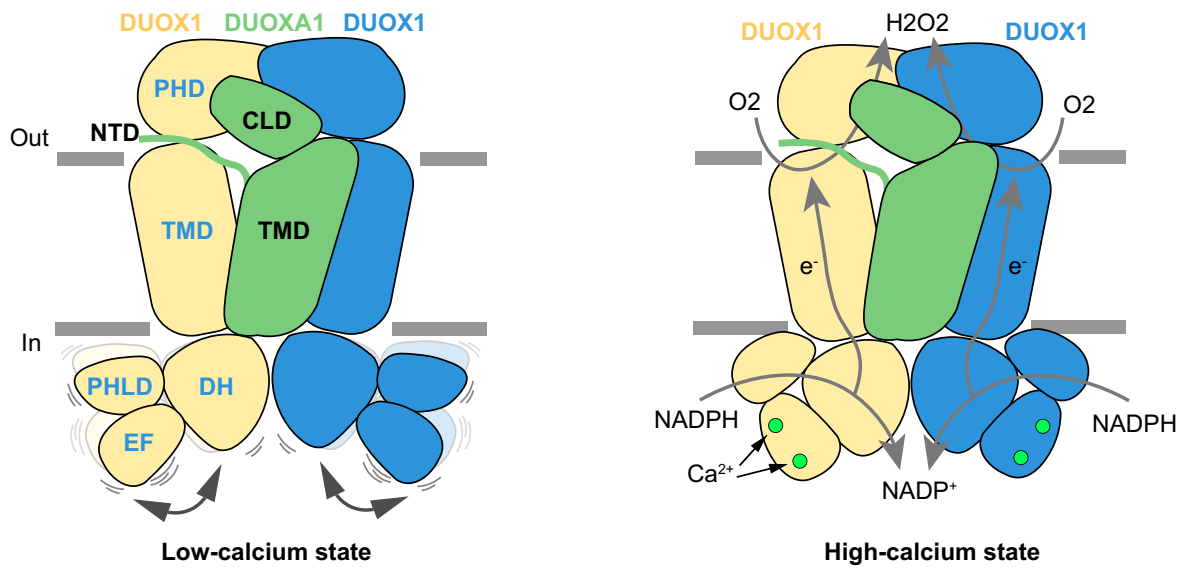

Fig. 6 Activation mechanism of DUOX1 complex by calcium. Two DUOX1 and one DUOXA1 subunit are shown as cartoon, and colored the same as Fig. 1d. Calcium ions are presented as green spheres. Electron transfer pathways are indicted with gray arrows.

reconfiguration of cytosolic inter-domain interactions which in turn mobilizes the DH domain and lowers the electron transfer efficiency (Fig. 6). These structures provide mechanistic insights into the structure and mechanism of DUOX and other NOX enzymes.

\section{Methods}

Cell culture. HEK293F suspension cells (Thermo Fisher Scientific) were cultured in Freestyle 293 medium (Thermo Fisher Scientific) supplemented with 1\% FBS at $37^{\circ} \mathrm{C}$ with $6 \% \mathrm{CO}_{2}$ and $70 \%$ humidity. Sf9 insect cells (Thermo Fisher Scientific) were cultured in SIM SF (Sino Biological) at $27^{\circ} \mathrm{C}$. The cell lines were routinely checked to be negative for mycoplasma contamination.

Protein expression and purification. We constructed a modified BacMam vector $^{28,29}$ with N-terminal GFP tag guided by rat FSH $\beta$ signal peptide ${ }^{10}$ and cloned hDUOX1 cDNA into this vector (please see Supplementary Table 2 for primer list). The hDUOX1 cDNA, we obtained from Prof. Han, has the same protein sequence as NP_059130.2 except the L1178F mutation, a SNP previously observed in AAI14939.1. The cDNAs of hDUOXA1 were cloned into a non-tagged BacMam vector or a modified BacMam vector with C-terminal MBP-mScarlet tag (please see Supplementary Table 2 for primer list) ${ }^{28,29}$. The hDUOX1 mutants were generated by Quick Change methods (please see Supplementary Table 2 for primer list). The two expression cassette were further merged into one bicistronic vector by the LINK sequence on the modified vector ${ }^{28,30}$. The baculoviruses were produced using the Bac-to-Bac system and amplified in Sf9 cells. For protein expression, HEK293F cells cultured in Freestyle 293 medium at density of $2.8 \times 10^{6} \mathrm{ml}^{-1}$ were infected with $15 \%$ volume of $\mathrm{P} 2$ virus. A total of $10 \mathrm{mM}$ sodium butyrate was added to the culture $12 \mathrm{~h}$ post infection and transferred to a $30^{\circ} \mathrm{C}$ incubator for another $36 \mathrm{~h}$ before harvesting. Cells were collected by centrifugation at $3999 \times g$ (JLA 8.1000, Beckman) for $10 \mathrm{~min}$, and washed with $20 \mathrm{mM}$ Tris (pH 8.0 at $4{ }^{\circ} \mathrm{C}$ ), $150 \mathrm{mM} \mathrm{NaCl}, 2 \mathrm{mM}$ EGTA, $2 \mu \mathrm{g} \mathrm{ml}^{-1}$ aprotinin, $2 \mu \mathrm{g} \mathrm{ml}{ }^{-1}$ pepstatin, $2 \mu \mathrm{g} \mathrm{ml}^{-1}$ leupeptin, flash-frozen, and storage at $-80^{\circ} \mathrm{C}$

For each batch of protein purification, cell pellet corresponding to 0.5 liter culture was thawed and extracted with $20 \mathrm{ml}$ buffer A $\left(20 \mathrm{mM}\right.$ Tris pH 8.0 at $4{ }^{\circ} \mathrm{C}$, $150 \mathrm{mM} \mathrm{NaCl}, 5 \mu \mathrm{g} \mathrm{ml}^{-1}$ aprotinin, $5 \mu \mathrm{g} \mathrm{ml}^{-1}$ pepstatin, $5 \mu \mathrm{g} \mathrm{ml}^{-1}$ leupeptin, $20 \%$ (v/v) glycerol, and $2 \mathrm{mM}$ EGTA) containing $1 \mathrm{mM}$ phenylmethanesulfonyl fluoride and $1 \%(\mathrm{w} / \mathrm{v})$ digitonin (Biosynth) at $4^{\circ} \mathrm{C}$ for $50 \mathrm{~min}$. A total of $1 \mathrm{mg} \mathrm{ml}^{-1}$ iodoacetamide (Sigma-I1149) was added during the detergent extraction procedure to reduce nonspecific cysteine crosslinking. The supernatant was ultracentrifuged at $135,300 \times g$ (TLA100.3, Beckman) for $50 \mathrm{~min}$. The solubilized proteins were loaded onto $5 \mathrm{ml}$ Streptactin Beads $4 \mathrm{FF}$ (Smart-Lifesciences) column and washed with $20 \mathrm{ml}$ buffer $\mathrm{A}+0.1 \%$ digitonin. The column was washed with $100 \mathrm{ml}$ buffer $\mathrm{A}+0.1 \%$ digitonin plus $10 \mathrm{mM} \mathrm{MgCl}_{2}$ and $1 \mathrm{mM}$ adenosine triphosphate (ATP) to remove contamination of heat shock proteins. Then the column was washed with $40 \mathrm{ml}$ buffer $\mathrm{A}+0.1 \%$ digitonin again to remove residual $\mathrm{MgCl}_{2}$ and ATP. The target protein was assembled into the peptidisc on the Streptactin Beads through washing with $4 \mathrm{ml}^{1} \mathrm{mg} \mathrm{ml}^{-1} \mathrm{NSPr}$ in $20 \mathrm{mM}$ Tris pH 8.0 (ref. ${ }^{12}$ ). Then the column was washed with $100 \mathrm{ml}$ buffer A to remove free NSPr. The assembled peptidiscs were eluted with $40 \mathrm{ml}$ buffer A $+5 \mathrm{mM} \mathrm{D}$ desthiobiotin (IBA). Eluted protein was concentrated using 100-kDa cutoff concentrator (Millipore) and further purified by Superose 6 increase (GE Healthcare) running in HBS (20 mM Hepes $\mathrm{pH} 7.5,150 \mathrm{mM} \mathrm{NaCl})+0.5 \mathrm{mM}$ EGTA. Fraction 19 corresponding to DUOX1 + DUOXA1 peptidisc complex was concentrated to $A 280 / 415=4.4 / 1.4$ with estimated concentration of $10.7 \mu \mathrm{M}$ DUOX1 subunits $\left(\varepsilon_{415}=0.131 \mu \mathrm{M}^{-1} \mathrm{~cm}^{-1}\right)$.
Enzymatic assay. The membrane fractions of DUOX1 for enzymatic assay were prepared as previously reported with minor modification ${ }^{31}$. Briefly, cells were washed with buffer A. After centrifuging at $3999 \times g$ for $10 \mathrm{~min}$ at $4{ }^{\circ} \mathrm{C}$, the cell pellets were broken using a needle for 12 times in $1 \mathrm{ml}$ of $20 \mathrm{mM}$ Tris pH 8.0 at $4{ }^{\circ} \mathrm{C}$ containing $0.1 \mathrm{mM}$ dithiothreitol, $10 \mathrm{mM}$ EGTA (pH 8.0), and the mixture of protease inhibitors. The pellet was removed by centrifuging at $3615 \times g$ for $15 \mathrm{~min}$, and the supernatant was collected and then centrifuged at $228,600 \times g$ (TLA100.3, Beckman) for $1 \mathrm{~h}$. The membrane pellet was resuspended in HBS containing $1 \mathrm{mM}$ EGTA. Meanwhile, $10 \mu \mathrm{l}$ membrane was solubilized by $100 \mu \mathrm{l}$ TBS $+1 \%$ digitonin with the mixture of protease inhibitors for $1 \mathrm{~h}$ at $4^{\circ} \mathrm{C}$ for FSEC. The protein concentrations in the membrane were estimated by comparing their GFP fluorescence signal to that of a purified GFP-tagged DUOX1 complex.

The $\mathrm{H}_{2} \mathrm{O}_{2}$-generating activity of DUOX1 complex was determined using the amplex red assay 32 . The concentrations of $\mathrm{H}_{2} \mathrm{O}_{2}$ solution were determined by measuring UV-vis absorbance at $240 \mathrm{~nm}$ with spectrophotometer (Pultton) and calculated using molar extinction coefficient of $43.6 \mathrm{M}^{-1} \mathrm{~cm}^{-1}$. The concentration of $\mathrm{H}_{2} \mathrm{O}_{2}$ solution was further validated by reacting with Amplex red to generate resorufin which has $\varepsilon_{571}=69,000 \mathrm{M}^{-1} \mathrm{~cm}^{-1}$ (ref. ${ }^{32}$ ). Then the $\mathrm{H}_{2} \mathrm{O}_{2}$ solution with known concentration was used to calibrate the resorufin fluorescence curve (excitation, $530 \mathrm{~nm}$; emission, $590 \mathrm{~nm}$ ) measured using a Microplate Reader (BioTek Synergy HT) at $37^{\circ} \mathrm{C}$.

The $\mathrm{H}_{2} \mathrm{O}_{2}$-generating reaction of the membrane fraction containing DUOX1 complex was performed at $37^{\circ} \mathrm{C}$ in $0.15 \mathrm{ml}$ of HBS with $1 \mathrm{mM}$ EGTA, $10 \mu \mathrm{M}$ FAD $100 \mu \mathrm{M}$ NADPH, $50 \mu \mathrm{M}$ amplex red, $0.067 \mathrm{mg} \mathrm{ml}^{-1}$ horseradish peroxidase, and $0.0576 \mathrm{mg} \mathrm{ml}^{-1}$ SOD. $\mathrm{Ca}^{2+}$ concentrations were determined using fluorescent indicators fura-2 or fluo3-FF. The $K_{\text {cat }}$ and $K_{\mathrm{m}}$ values of the membrane fraction containing DUOX1 complex were determined at $37^{\circ} \mathrm{C}$ with different concentrations of NADPH in the presence or absence of $1.4 \mathrm{mM} \mathrm{CaCl}_{2}$. The $\mathrm{H}_{2} \mathrm{O}_{2}$ generating reaction of the purified DUOX1 complex in peptidisc was performed at $27^{\circ} \mathrm{C}$ in $0.15 \mathrm{ml}$ of $\mathrm{HBS}+1 \mathrm{mM}$ EGTA, $10 \mu \mathrm{M} \mathrm{FAD}, 100 \mu \mathrm{M} \mathrm{NADPH}, 50 \mu \mathrm{M}$ amplex red, $0.067 \mathrm{mg} \mathrm{ml}^{-1}$ horseradish peroxidase, and $0.0576 \mathrm{mg} \mathrm{ml}^{-1} \mathrm{SOD}$ in the presence or absence of $1.1 \mathrm{mM} \mathrm{CaCl}_{2}$. Progress of the reactions was monitored continuously by following the increase of the resorufin fluorescence, and the initial reaction rates were obtained by fitting the curve with linear equation. The activity of DUOX1 complex was determined by subtracting the background of the corresponding buffer without enzyme. The data were processed with Microsoft Excel-2013, SigmaPlot-12.0, and GraphPad Prism 6.

Cryo-EM sample preparation and data acquisition. The peptidisc sample was supplemented with $2.5 \mathrm{mM}$ EGTA (low calcium) or $0.5 \mathrm{mM}$ free calcium (high calcium) for cryo-EM analysis, respectively. Both samples contain $100 \mu \mathrm{M}$ FAD as the cofactor and $500 \mu \mathrm{M} \mathrm{NADPH}$ as the substrate. To overcome the preferred orientation problem, $0.5 \mathrm{mM}$ non-solubilizing detergent fluorinated octyl-maltoside was added to the sample before cyro-EM sample preparation. Aliquots of $1.5 \mu \mathrm{L}$ protein sample were placed on graphene oxide-coated grids, as previously reported ${ }^{33}$. Grids were blotted for $3 \mathrm{~s}$ at $100 \%$ humidity and flash-frozen in liquid ethane cooled by liquid nitrogen using Vitrobot Mark I (FEI). Grids were then transferred to a Titan Krios (FEI) electron microscope that was equipped with a Gatan GIF Quantum energy filter and operated at $300 \mathrm{kV}$ accelerating voltage. Image stacks were recorded on a Gatan K2 Summit direct detector in super-resolution counting mode using Serial EM at a nominal magnification of $130,000 \times$ (calibrated pixel size of $1.045 \AA$ pixel $^{-1}$ ), with a defocus ranging from -1.5 to $-2.0 \mu \mathrm{m}$. Each stack of 32 frames was exposed for $7.12 \mathrm{~s}$, with a total dose $\sim 50 \mathrm{e}^{-} \AA^{-2}$ and a dose rate of $8 \mathrm{e}^{-}$pixel $^{-1} \mathrm{~s}^{-1}$ on detector.

Image processing. The image processing workflow is illustrated in Figs. S2 and S4. A total of 7076 super-resolution movie stacks of the high-calcium state sample and 
2076 stacks of the low-calcium state sample were collected using Serial EM, and motion-corrected, dose weighted, and twofold binned to a pixel size of $1.045 \AA$ using MotionCor2 (ref. ${ }^{34}$ ). Contrast transfer function (CTF) parameters were estimated with Gctf ${ }^{35}$. Micrographs with ice or ethane contamination, and empty carbon were removed manually. Autopicking were performed using Gautomatch (kindly provided by Kai Zhang). All subsequent classification and reconstruction was performed in Relion 3.1 (ref. ${ }^{36}$ ) unless otherwise stated. Reference-free 2D classification was performed to remove contaminants. Initial model was generated using cryoSPARC ${ }^{37}$. Particles were subjected to multi-reference 3D classification $^{38,39}$ and random-phase $3 \mathrm{D}$ classification ${ }^{38,39}$. Phase-randomized models were generated from the model obtained from previous refinement using randomize software (from the lab of Nikolaus Grigorieff). Further CTF refinement was then performed with Relion 3.1 using C2 symmetry. The particles were then re-extracted, re-centered, and re-boxed from 256 pixels to 320 pixels for consensus refinement in Relion 3.1 (ref. ${ }^{36}$ ) and cryoSPARC ${ }^{37}$. To improve the density of cytosolic layer, particles were symmetry expended ${ }^{13}$ for multibody refinement ${ }^{14}$. One soft mask (the large body) that covers the extracellular domain together with TMD of one protomer was generated from the consensus map, using UCSF Chimera and Relion 3.1 (ref. ${ }^{36}$ ). The other soft mask (the small body) covers the cytosolic domains of the same protomer. 3D multibody refinements ${ }^{14}$ were performed using the two soft masks and the parameters determined from previous consensus refinement. The motions of the bodies were analyzed by relion_flex analyse in Relion 3.1 (ref. ${ }^{36}$ ). The two half-maps of each body generated by 3D multibody refinement were subjected to post-processing in Relion 3.1 (ref. ${ }^{36}$ ). The masked and sharpened maps of each body were aligned to the consensus map using UCSF Chimera ${ }^{40}$, zoned to isolated nonoverlapping regions and summed using Relion 3.1 (ref. ${ }^{36}$ ) to generate the composite maps for visualization and model building. All of the resolution estimations were based on a Fourier shell correlation of 0.143 cutoff after correction of the masking effect. $B$-factor used for map sharpening was automatically determined by the post-processing procedure in Relion 3.1 (ref. ${ }^{36}$ ). The local resolution was estimated with Relion 3.1 (ref. ${ }^{36}$ ).

Model building. The composite maps derived from multibody refinement were used for model building. The structures of PHD, TMD, EF1-2, and DH domains of hDUOX1 were generated using phyre2 server ${ }^{41}$ based on PDB ID: 6ERC, 5O0T, $4 \mathrm{LL} 1$, and 5O0X, and manually docked into the cryo-EM maps using Chimera ${ }^{40}$. Initial models of PHLD were generated by Rosetta Web Server using ab initio mode ${ }^{42}$, manually selected according to the distances calculated by RaptorX Contact Prediction server ${ }^{43}$, and validated by the fitting between model and cryoEM densities, especially the location of bulky aromatic residues. The partial model of hDUOXA1 were generated using EM builder ${ }^{44}$. The initial models were iteratively built using $\operatorname{Coot}^{45}$ and refined using Phenix in real space ${ }^{46}$. Figures were prepared using UCSF chimera ${ }^{40}$, Chimera $\mathrm{X}^{47}$, and Pymol.

Reporting summary. Further information on research design is available in the Nature Research Reporting Summary linked to this article.

\section{Data availability}

Data supporting the findings of this manuscript are available from the corresponding author upon reasonable request. A reporting summary for this article is available as a Supplementary Information file. The cryo-EM map of DUOX1-DUOXA1 in the highcalcium and low-calcium states have been deposited in the EMDB under ID code EMD30556 and EMD-30555. The atomic coordinate of DUOX1-DUOXA1 in the highcalcium and low-calcium states have been deposited in the PDB under ID code 7D3F and 7D3E, respectively. The following PDB entries used in this study were downloaded from Protein Data Bank: 6ERC, 5O0T, 4IL1, 5O0X, 1QFZ, 6OV2, 3A1F, and 3KZ1. The cryoEM map of mouse DUOX1-DUOXA1 used in this study was downloaded from the EMDB under ID code EMD-21964. Source data are provided with this paper.

Received: 6 October 2020; Accepted: 2 December 2020;

Published online: 08 January 2021

\section{References}

1. Sies, H. \& Jones, D. P. Reactive oxygen species (ROS) as pleiotropic physiological signalling agents. Nat. Rev. Mol. Cell Biol. 21, 363-383 (2020).

2. Lambeth, J. D. \& Neish, A. S. Nox enzymes and new thinking on reactive oxygen: a double-edged sword revisited. Annu. Rev. Pathol. 9, 119-145 (2014).

3. Winterbourn, C. C., Kettle, A. J. \& Hampton, M. B. Reactive oxygen species and neutrophil function. Annu. Rev. Biochem. 85, 765-792 (2016).

4. Ohye, H. \& Sugawara, M. Dual oxidase, hydrogen peroxide and thyroid diseases. Exp. Biol. Med. 235, 424-433 (2010).

5. Grasberger, H. \& Refetoff, S. Identification of the maturation factor for dual oxidase. Evolution of an eukaryotic operon equivalent. J. Biol. Chem. 281, 18269-18272 (2006).
6. Weber, G., Rabbiosi, S., Zamproni, I. \& Fugazzola, L. Genetic defects of hydrogen peroxide generation in the thyroid gland. J. Endocrinol. Investig. 36, 261-266 (2013)

7. Sumimoto, H. Structure, regulation and evolution of Nox-family NADPH oxidases that produce reactive oxygen species. FEBS J. 275, 3249-3277 (2008).

8. Karplus, P. A., Daniels, M. J. \& Herriott, J. R. Atomic structure of ferredoxin$\mathrm{NADP}^{+}$reductase: prototype for a structurally novel flavoenzyme family. Science 251, 60-66 (1991).

9. Magnani, F. et al. Crystal structures and atomic model of NADPH oxidase. Proc. Natl Acad. Sci. USA 114, 6764-6769 (2017).

10. Long, D. et al. New insight into the mechanism underlying fibroin secretion in silkworm, Bombyx mori. FEBS J. 282, 89-101 (2015).

11. Forteza, R., Salathe, M., Miot, F. \& Conner, G. E. Regulated hydrogen peroxide production by Duox in human airway epithelial cells. Am. J. Respir. Cell Mol. Biol. 32, 462-469 (2005).

12. Carlson, M. L. et al. The Peptidisc, a simple method for stabilizing membrane proteins in detergent-free solution. Elife 7, e34085 (2018).

13. Zhou, M. et al. Atomic structure of the apoptosome: mechanism of cytochrome c- and dATP-mediated activation of Apaf-1. Genes Dev. 29, 2349-2361 (2015).

14. Nakane, T., Kimanius, D., Lindahl, E. \& Scheres, S. H. Characterisation of molecular motions in cryo-EM single-particle data by multi-body refinement in RELION. elife 7, e36861 (2018).

15. Nicolussi, A. et al. Secreted heme peroxidase from Dictyostelium discoideum: insights into catalysis, structure, and biological role. J. Biol. Chem. 293, 1330-1345 (2018)

16. Holm, L. Benchmarking fold detection by DaliLite v.5. Bioinformatics 35, 5326-5327 (2019).

17. Feng, J., He, L., Li, Y., Xiao, F. \& Hu, G. Modeling of PH domains and phosphoinositides interactions and beyond. Adv. Exp. Med. Biol. 1111, 19-32 (2019).

18. Ye, Q. et al. Structural basis of calcineurin activation by calmodulin. Cell. Signal. 25, 2661-2667 (2013).

19. Rigutto, S. et al. Activation of dual oxidases Duox1 and Duox2: differential regulation mediated by camp-dependent protein kinase and protein kinase Cdependent phosphorylation. J. Biol. Chem. 284, 6725-6734 (2009).

20. Peters, C. et al. DUOX2/DUOXA2 mutations frequently cause congenital hypothyroidism that evades detection on newborn screening in the United Kingdom. Thyroid 29, 790-801 (2019).

21. Deng, Z. et al. A productive NADP + binding mode of ferredoxin-NADP + reductase revealed by protein engineering and crystallographic studies. Nat. Struct. Biol. 6, 847-853 (1999).

22. Chovancova, E. et al. CAVER 3.0: a tool for the analysis of transport pathways in dynamic protein structures. PLoS Comput. Biol. 8, el002708 (2012).

23. Ueyama, T. et al. The extracellular A-loop of dual oxidases affects the specificity of reactive oxygen species release. J. Biol. Chem. 290, 6495-6506 (2015).

24. Morand, S. et al. Duox maturation factors form cell surface complexes with Duox affecting the specificity of reactive oxygen species generation. FASEB J. 23, 1205-1218 (2009).

25. Hoste, C., Dumont, J. E., Miot, F. \& De Deken, X. The type of DUOXdependent ROS production is dictated by defined sequences in DUOXA. Exp. Cell Res. 318, 2353-2364 (2012)

26. Vecchio, A. J. \& Stroud, R. M. Claudin-9 structures reveal mechanism for toxin-induced gut barrier breakdown. Proc. Natl Acad. Sci. USA 116, 17817-17824 (2019).

27. Sun, J. Structures of mouse DUOX1-DUOXA1 provide mechanistic insights into enzyme activation and regulation. Nat. Struct. Mol. Biol. 27, 1086-1093 (2020).

28. Li, N. et al. Structure of a pancreatic ATP-sensitive potassium channel. Cell 168, 101-110 e110 (2017).

29. Goehring, A. et al. Screening and large-scale expression of membrane proteins in mammalian cells for structural studies. Nat. Protoc. 9, 2574-2585 (2014).

30. Scheich, C., Kummel, D., Soumailakakis, D., Heinemann, U. \& Bussow, K. Vectors for co-expression of an unrestricted number of proteins. Nucleic Acids Res. 35, e43 (2007).

31. Ameziane-El-Hassani, R. et al. Dual oxidase-2 has an intrinsic $\mathrm{Ca} 2^{+}-$ dependent H2O2-generating activity. J. Biol. Chem. 280, 30046-30054 (2005).

32. Zhou, M., Diwu, Z., Panchuk-Voloshina, N. \& Haugland, R. P. A stable nonfluorescent derivative of resorufin for the fluorometric determination of trace hydrogen peroxide: applications in detecting the activity of phagocyte NADPH oxidase and other oxidases. Anal. Biochem 253, 162-168 (1997).

33. Phulera, S. et al. Cryo-EM structure of the benzodiazepine-sensitive alphalbetalgamma2S tri-heteromeric GABAA receptor in complex with GABA. eLife 7, e39383 (2018).

34. Zheng, S. Q. et al. MotionCor2: anisotropic correction of beam-induced motion for improved cryo-electron microscopy. Nat. Methods 14, 331-332 (2017). 
35. Zhang, K. Gctf: real-time CTF determination and correction. J. Struct. Biol. 193, 1-12 (2016).

36. Zivanov, J. et al. New tools for automated high-resolution cryo-EM structure determination in RELION-3. Elife 7, e42166 (2018).

37. Punjani, A., Rubinstein, J. L., Fleet, D. J. \& Brubaker, M. A. cryoSPARC: algorithms for rapid unsupervised cryo-EM structure determination. Nat. Methods 14, 290-296 (2017).

38. Kang, Y., Wu, J.-X. \& Chen, L. Structure of voltage-modulated sodiumselective NALCN-FAM155A channel complex. Nat. Commun. 11, 6199 (2020).

39. Gong, X. et al. Structural insights into the Niemann-Pick C1 (NPC1)mediated cholesterol transfer and ebola infection. Cell 165, 1467-1478 (2016).

40. Pettersen, E. F. et al. UCSF Chimera-a visualization system for exploratory research and analysis. J. Comput Chem. 25, 1605-1612 (2004).

41. Kelley, L. A., Mezulis, S., Yates, C. M., Wass, M. N. \& Sternberg, M. J. The Phyre2 web portal for protein modeling, prediction and analysis. Nat. Protoc. 10, 845-858 (2015).

42. Raman, S. et al. Structure prediction for CASP8 with all-atom refinement using Rosetta. Proteins 77, 89-99 (2009).

43. Wang, S., Sun, S., Li, Z., Zhang, R. \& Xu, J. Accurate de novo prediction of protein contact map by ultra-deep learning model. PLoS Comput. Biol. 13, e1005324 (2017).

44. Zhou, N., Wang, H. \& Wang, J. EMBuilder: a template matching-based automatic model-building program for high-resolution cryo-electron microscopy maps. Sci. Rep. 7, 2664 (2017).

45. Emsley, P., Lohkamp, B., Scott, W. G. \& Cowtan, K. Features and development of Coot. Acta Crystallogr. D Biol. Crystallogr. 66, 486-501 (2010).

46. Afonine, P. V. et al. Real-space refinement in PHENIX for cryo-EM and crystallography. Acta Crystallogr. D. Struct. Biol. 74, 531-544 (2018).

47. Pettersen, E. F. et al. UCSF ChimeraX: structure visualization for researchers, educators, and developers. Protein Sci. https://doi.org/10.1002/pro.3943 (2020). Online ahead of print.

\section{Acknowledgements}

We thank Yunlu Kang, Wenjun Guo, Yange Niu, Dian Ding, Mengmeng Wang, Miao Wei, and Xiao He for illustration. We thank Miao Wei for making the rat FSH $\beta$ secretion signal-guided GFP vector. We thank Prof. Jiahuai Han for providing the cDNA of hDUOX1 and hDUOXA1, Prof. Helmut Grasberger for providing the cDNA of hDUOX2 and hDUOXA2, Prof. Yuji Kohara for providing the cDNA of Caenorhabditis elegans duox-2, bli-3, and doxA1. We thank the National Center for Protein Sciences at Peking University in Beijing, China for assistance with negative-stain EM. Cryo-EM data collection was supported by Electron microscopy laboratory and Cryo-EM platform of Peking University with the assistance of Xuemei Li, Daqi Yu, Xia Pei, Bo Shao, Guopeng Wang, and Zhenxi Guo. We thank the National Center for Protein Sciences at Peking University in Beijing, China for assistance with negative-stain EM. Part of structural computation was also performed on the Computing Platform of the Center for Life Science and High-performance Computing Platform of Peking University. This work is supported by grants from the Ministry of Science and Technology of China (National
Key R\&D Program of China, 2016YFA0502004 to L.C.), National Natural Science Foundation of China (91957201, 31870833, and 31821091 to L.C., and 31900859 to J.-X. W.), Beijing Natural Science Foundation (5192009 to L.C.), and Young Thousand Talents Program of China to L.C., and the China Postdoctoral Science Foundation (2016M600856, 2017T100014, 2019M650324, and 2019T120014 to J.-X.W.). J.-X.W. is supported by the Boya Postdoctoral Fellowship of Peking University and the postdoctoral foundation of the Peking-Tsinghua Center for Life Sciences, Peking University (CLS).

\section{Author contributions}

L.C. initiated the project. J.-X.W. purified proteins and prepared the cryo-EM samples, collected the cryo-EM data, and processed the cryo-EM data. L.C. built and refined the atomic model. R.L. and K.S. screened NOX constructs. All authors contributed to the manuscript preparation.

\section{Competing interests}

The authors declare no competing interests.

\section{Additional information}

Supplementary information is available for this paper at https://doi.org/10.1038/s41467020-20466-9.

Correspondence and requests for materials should be addressed to L.C.

Peer review information Nature Communications thanks Andrea Mattevi and other, anonymous, reviewers for their contributions to the peer review of this work. Peer review reports are available.

Reprints and permission information is available at http://www.nature.com/reprints Publisher's note Springer Nature remains neutral with regard to jurisdictional claims in published maps and institutional affiliations.

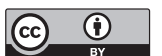

Open Access This article is licensed under a Creative Commons Attribution 4.0 International License, which permits use, sharing, adaptation, distribution and reproduction in any medium or format, as long as you give appropriate credit to the original author(s) and the source, provide a link to the Creative Commons license, and indicate if changes were made. The images or other third party material in this article are included in the article's Creative Commons license, unless indicated otherwise in a credit line to the material. If material is not included in the article's Creative Commons license and your intended use is not permitted by statutory regulation or exceeds the permitted use, you will need to obtain permission directly from the copyright holder. To view a copy of this license, visit http://creativecommons.org/ licenses/by/4.0/.

(C) The Author(s) 2021 